\title{
Mehr Mut zur Reform
}

\author{
Stefan G. Spitzer
}

Online publiziert: 20. April 2011

(C) Springer-Verlag 2011

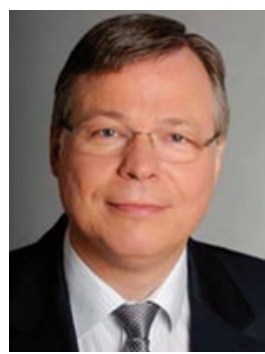

Daseinsvorsorge in einer, „Gesellschaft des längeren Lebens“. Mit kleinen Effekten wird uns da nicht geholfen sein.

Also kann doch die Frage nur lauten: Wodurch erhöhen wir deutlich die Effizienz der Versorgung? Die nicht geringen Reserven, die in unserem Gesundheitswesen noch erschlossen werden können, sind doch bekannt. Eine solche Reform zu wagen bedeutet auch, nicht allen Gruppeninteressen gerecht werden zu können.

Wir brauchen dazu nicht nur fähige Köpfe, sondern auch

Stefan G. Spitzer

Dieses Jahr ist - wieder einmal - ein wichtiges Jahr. Bedeutsame politische Wahlen wollen überstanden sein. Da steigt der Pegel der Empfindsamkeit bei den politischen Parteien. Nur nichts falsch machen, nichts riskieren, man braucht die Stimmen der Wähler...

Und ausgerechnet in diesem Jahr soll der große Wurf gelingen: die Reform der GKV-Versorgung. Zunächst sind sich alle einig: Große Aufgaben stehen vor uns, so darf es nicht weitergehen, vieles muss geändert werden.

Das war's dann aber auch schon mit der Einigkeit. Neue Begriffe kursieren, „sektorenübergreifende Bedarfsplanung“, ,regionaler Versorgungsausschuss“, ,gleiche Wettbewerbschancen für ambulant und stationär"... und jeder hat davon eigene Vorstellungen. Mit hoher Geschwindigkeit werden Absichten formuliert und wieder verworfen, strategische Allianzen geschmiedet und wieder gelöst.

Gewiss - es geht um viel. Aber die Versorgungsreform muss auch viel bewirken. Die Gelder werden knapp für die

Prof. Dr. med. S. G. Spitzer $(\bowtie)$

Praxisklinik Herz und Gefäße,

Akad. Lehrpraxisklinik der TU Dresden,

Forststraße 3, 01099 Dresden, Deutschland

E-Mail: prof.spitzer@praxisklinik-dresden.de mehr Mut zu großen Entscheidungen. Sonst könnte es Ende des Jahres heißen: „Der Berg kreißte...“.

Vom 19. bis 20. November 2010 fand in Dresden das 16. Dresdner Symposium „Herz und Gefäße - Innovationen 2010“ statt. Der enorme wissenschaftlich-technische Fortschritt in der Herz-Kreislauf-Medizin und die sich daraus ergebenden Verbesserungen in Diagnostik und Therapie bieten Jahr für Jahr eine Fülle an zu diskutierenden Themen.

Am Anfang des Supplementbandes steht der Übersichtsartikel zur Geschichte der Herzinsuffizienz von Prof. Lüderitz, der dieses Thema in gewohnt exzellenter Art und Weise vorstellt.

Im Weiteren wird ein Überblick zur Diagnostik und Therapie des Diabetes mellitus sowie von Fettstoffwechselstörungen gegeben; weitere Arbeiten widmen sich der intermediären Ischämiediagnostik unter Einschluss neuester Diagnostikmethoden wie dem Cardiac Hybrid Imaging.

Nachfolgend werden die aktuellen Daten der Syntax-Studie diskutiert sowie Technik und Ergebnisse des transapikalen Aortenklappenersatzes vorgestellt.

Den Abschluss des Heftes bilden Arbeiten zur Antikoagulation bei Vorhofflimmern sowie zu neuen Technologien bei der Katheterablation. 